Revue européenne des sciences sociales

European Journal of Social Sciences

57-1 | 2019

Présence de Max Weber, la portée d'un classique

\title{
Louis PINTO, L'Invention du consommateur. Sur la légitimité du marché
}

\section{Simon Langlois}

\section{(2) OpenEdition}

1 Journals

\section{Édition électronique}

URL : https://journals.openedition.org/ress/4801

DOI : 10.4000/ress.4801

ISBN : $1663-4446$

ISSN : 1663-4446

Éditeur

Librairie Droz

Édition imprimée

Date de publication : 30 mai 2019

Pagination : 303-305

ISSN : 0048-8046

Référence électronique

Simon Langlois, «Louis PINTO, L'Invention du consommateur. Sur la légitimité du marché ", Revue européenne des sciences sociales [En ligne], 57-1 | 2019, mis en ligne le 21 mars 2019, consulté le 05 janvier 2023. URL : http://journals.openedition.org/ress/4801 ; DOI : https://doi.org/10.4000/ress. 4801

Ce document a été généré automatiquement le 5 janvier 2023.

Tous droits réservés 


\title{
Louis PINTO, L'Invention du consommateur. Sur la légitimité du marché
}

\author{
Simon Langlois
}

\section{RÉFÉRENCE}

Louis PINTO, 2018, L'Invention du consommateur. Sur la légitimité du marché, Paris, PUF,

«Le lien social », 286 p.

1 Louis Pinto propose une synthèse de plusieurs travaux empiriques réalisés depuis les années 1990 sur la consommation marchande et sur l'émergence ou la généalogie de la figure du consommateur.

2 L'ouvrage comprend deux parties. La première porte sur l'interprétation de la société de consommation et en particulier sur les discours critiques dont elle a été l'objet par différents essayistes dans les années 1960. Pinto montre bien comment ces essais ont objectivé la catégorie de consommateur qui était alors vu comme étant manipulé et aliéné, berné aussi par la perspective du bonheur consumériste. L'auteur précise avec justesse que ces analyses critiques ne s'appuyaient pas sur des études empiriques, une lacune qu'il a contribué à combler par ses propres travaux sur lesquels il a basé les chapitres suivants de son livre.

Pinto évoque avec une grande érudition la manière dont les différentes luttes au nom du consommateur ont contribué à imposer une "définition légitime » de ce dernier, à distance du discours critique et politisé. Deux couples d'opposition vont émerger avec l'extension de la consommation marchande. Le couple individu aliéné / individu révolté côtoie désormais le couple individu vigilant / individu ignorant, celui-ci ayant donné naissance à une vision dépolitisée de la consommation. Pour l'auteur, ce dernier couple s'inscrit dans la gestion libérale des problèmes de consommation mais il est coextensif 
à l'action des militants et à l'avènement d'une « nébuleuse d'organisations » qui ont fait du consommateur un véritable acteur social.

4 L'émergence de la définition légitime du consommateur et la nouvelle action militante qui le constitue comme acteur social nécessitaient un travail d'institutionnalisation, objet de la seconde partie de son ouvrage, la plus originale et celle qui justifie le titre du livre. «Il est nécessaire de comprendre de quelle façon la catégorie de consommateur a été dotée d'une force sociale effective qui a eu des effets sur le réel : loin d'être un mot ou une simple "représentation", la faculté de choisir de l'individu autonome et souverain a été construite par l'État, le droit, des groupes professionnels, des associations» (p.16). L'institutionnalisation de la catégorie de consommateur est analysée à travers trois cas de figure. Le consommateur a été considéré comme label politique dans la sphère politico-administrative, défini comme figure juridique par le droit et enfin inscrit comme client dans la relation marchande.

Pinto montre d'abord comment s'affrontent les pouvoirs publics, les organisations de consommateurs et les détenteurs du pouvoir économique autour du statut à donner au consommateur. Ces trois entités sont en conflit ou du moins en concurrence afin de définir ce dernier. Par exemple, les entreprises peuvent difficilement ne pas considérer leurs intérêts économiques en faisant valoir les besoins des consommateurs, alors que les organisations de consommateurs ont de la difficulté à établir leur représentativité, leur dispersion affaiblissant les prétentions de chacune d'entre elles.

6 L'auteur analyse ensuite comment le droit naturalise la catégorie de consommateur qui devient une figure juridique. Le droit des obligations, élément du droit civil français, n'était pas adapté à la consommation marchande élargie. Au couple traditionnel de l'acheteur et du vendeur, le droit de la consommation a fait émerger une nouvelle figure, celle du professionnel, "figure en laquelle se trouvent réunies celles de commerçant et de fabriquant» (p. 222). «Le consommateur s'est vu défini par le droit selon deux logiques en partie divergentes, mais appelées à se combiner, à la fois comme co-contractant dans l'esprit du code civil et comme un acheteur de biens et services inséré dans un ordre public économique, le marché concurrentiel» (p. 237). Devenu consommateur, l'acteur social conserve sa faculté de choisir mais elle est encadrée par un dispositif institutionnel construit par le droit.

7 Enfin, Pinto avance que le consommateur est devenu un client dans la relation marchande désormais dotée de civilité. Une sorte de tiers exclus s'est imposé entre la pure violence (le vendeur fraude) et le pur altruisme (le vendeur veut avant tout satisfaire le client). "Tout se passe comme si la confrontation entre acheteur et vendeur avait été insérée dans un ensemble de règles rendant possible la pacification de cette relation » (p. 263). Si le vendeur entend bien pousser le client à l'achat, il doit le faire «dans des formes socialement licites, parfois coûteuses, qui permettent de garantir la souveraineté du client promu à la dignité de consommateur » (p. 264).

Dans sa vision de la consommation marchande, Pinto s'inspire de la perspective mise de l'avant par Pierre Bourdieu. Les pratiques de consommation sont liées les unes aux autres par un principe unitaire qui est celui du style de vie. "À la relation intuitivement perçue entre un sujet consommateur et un objet consommé, on doit substituer la correspondance systématique entre deux espaces, celui des styles de vie et celui des produits, celui des agents classants et celui des biens classés. Les comportements ne sont pas séparables des modes de classement et d'évaluation et, en outre, ceux-ci ne sont pas dissociables de leurs conditions économiques de possibilité » 
(p. 267). Cependant, cette sociologie de la consommation paraît trop rigide de nos jours, teintée d'un certain déterminisme qui a maintes fois été reprochée à la perspective bourdieusienne. Par contre, il ne fait aucun doute que la consécration du consommateur émerge du droit, des interventions de l'état législateur en matière de consommation ainsi que de l'action des associations parlant au nom des consommateurs. Si l'analyse de Pinto est convaincante et bien appuyée empiriquement, elle me semble incomplète pour deux raisons.

9 Tout d'abord la hausse réelle du niveau de vie des ménages, l'avènement du revenu discrétionnaire et l'extension de la scolarisation ont étendu la marge de manœuvre des acteurs sociaux et leur espace de liberté. Les pesanteurs sociales d'autrefois, la faiblesse des ressources monétaires et les contraintes morales sont moins fortes pour une partie non négligeable de la population et moins déterminantes qu'à l'époque des enquêtes budgétaires de Maurice Halbwachs. Les « systèmes de relations » évoqués par Bourdieu sont par conséquent devenus plus ouverts, plus diversifiés, ce qui n'est pas sans se refléter dans les patrons de consommations. Ainsi, les consommateurs au sommet de la hiérarchie sociale ne sont-ils pas devenus omnivores, au sens donné à ce terme par Richard Peterson. De même, les générations qui se succèdent - plus scolarisées, avec des revenus plus élevés - contribuent-elles à brouiller le principe unitaire des styles de vie.

10 Par ailleurs, la multiplicité des objets de consommation (biens ou services) leur donne un pouvoir structurant, rendant possible un large éventail de comportements moins déterminés qu'autrefois. Pensons seulement aux consommations culturelles et aux pratiques de l'information avec l'avènement d'Internet. L'offre est devenue tellement étendue qu'elle rend possible l'émergence de configurations comportementales très diversifiées et l'avènement de ce que plusieurs sociologues appellent «la société des individus ».

11 L'analyse de Pinto a le mérite de montrer que l'action du consommateur s'inscrit dans un système économique contraignant. "Le choix souverain du consommateur doit certes être célébré, mais seulement pour autant qu'il n'entre pas en contradiction avec les objectifs de la politique économique et avec les stratégies des entreprises » (p. 269), avance-t-il en conclusion. Il importait de le rappeler.

\section{AUTEURS}

\section{SIMON LANGLOIS}

Université Laval (Québec) - Faculté des sciences sociales, Département de sociologie 